\title{
Pipe Flow of Suspensions Containing Bubbles
}

\author{
Katharina Gladbach1, Antonio Delgado1, Cornelia Rauh ${ }^{1,2}$ \\ ${ }^{1}$ Institute of Fluid Mechanics, Friedrich-Alexander-University Erlangen-Nuremberg, Erlangen, Germany \\ ${ }^{2}$ Institute of Food Technology and Food Chemistry, Technical University Berlin, Berlin, Germany \\ Email: katharina.gladbach@gmx.de
}

How to cite this paper: Gladbach, K., Delgado, A. and Rauh, C. (2018) Pipe Flow of Suspensions Containing Bubbles. World Journal of Mechanics, 8, 417-429 https://doi.org/10.4236/wjm.2018.810030

Received: October 6, 2018

Accepted: October 27, 2018

Published: October 30, 2018

Copyright $\odot 2018$ by authors and Scientific Research Publishing Inc. This work is licensed under the Creative Commons Attribution International License (CC BY 4.0).

http://creativecommons.org/licenses/by/4.0/ (c) (i) Open Access

\begin{abstract}
The steady laminar pipe flow of a suspension with a gas volume fraction $\phi \leq$ 0.5 and small or intermediate bubble deformations in long, and straight sections of a circular pipe is calculated. The calculations are based on the constitutive equation that was originally derived for dilute emulsions and further developed for concentrated suspensions containing bubbles. In contrast to the literature, an analytical procedure is used to determine the solution of a pipe flow more accurately. The results are presented and discussed with respect to the Reynolds number Re and capillary number $\mathrm{Ca}$. If $\mathrm{Ca}<1$ or $\mathrm{Ca}>$ 1 , a bubble suspension has a parabolic velocity profile indicating a Newtonian rheology. If $\mathrm{Ca} \approx 1$, two regimes of flow are observed in agreement with the literature; that is, an inner plug flow where deformation rates are low and an outer flow where deformation rates are high. These results imply that, if $\mathrm{Ca}<$ 1, the Reynolds number Re decreases with increasing gas volume fraction $\phi$ and that, if $\mathrm{Ca} \geq 1$, the opposite effect occurs; that is, the Reynolds number $\mathrm{Re}$ increases with increasing gas volume fraction.
\end{abstract}

\section{Keywords}

Pipe Flow, Suspension of Bubbles, Constitutive Equation, Capillary Number

\section{Introduction}

Foams become more important in industrial applications, but also in food production because of many advantages, including low weight, thermal insulation and special texture characteristics. Relevant constitutive equations were developed by Oldroyd [1] [2] [3] [4] and by Frankel and Acrivos [5] [6]. They studied the rheological behavior of dilute emulsions in steady or weakly time-dependent flows. The models have also been used to describe the rheological behavior of dilute bubble suspensions. Contributions were provided by Llewellin, Mader and Wilson [7] [8], by Llewellin and Manga [9] and by Pal [10] [11] among others. 
For bubble suspensions, where the ratio of the dispersed-phase viscosity to the continuous-phase viscosity is essentially zero, the viscosity ratio is simply set equal to zero. The constitutive equations derived by Oldroyd [1] [2] [3] and by Frankel and Acrivos [5] [6] are only valid for small bubble deformations. Llewellin, Mader, Wilson and Manga [7] [8] [9] and Pal [10] [11], however, also applied the analytical equations (as approximation) when high capillary numbers occur, provided that the bubbles do not rupture. In this case, the rheological equations do not present analytical equations anymore, but they become empirical expressions adapted to the experimental data that were used by the authors [7]-[16]. In the following, the constitutive equation derived by Oldroyd [2] for dilute emulsions and further developed by Pal [11] for concentrated suspensions of bubbles is presented. Subsequently, the analytical procedure to calculate a pipe flow of a two-phase system is explained. The results are presented and discussed with respect to the Reynolds number Re and capillary number Ca.

\section{Model for Suspensions Containing Bubbles}

Oldroyd [1] [2] [3] developed a constitutive equation for a dilute emulsion consisting of two incompressible Newtonian liquids. The model is subject to the restrictions that inertia effects and hydrodynamic interactions between drops are ignored and drop deformations are very small. Oldroyd obtained a linear relation between the extra stress tensor $S$, the deformation velocity tensor $E$ and their time derivatives. For bubble suspensions, the constitutive equation becomes

$$
\left(\boldsymbol{S}+\lambda_{1} \frac{\mathcal{D} \boldsymbol{S}}{\mathcal{D} t}\right)=2 \eta_{0}\left(\boldsymbol{E}+\lambda_{2} \frac{\mathcal{D} \boldsymbol{E}}{\mathcal{D} t}\right) .
$$

$\mathcal{D} / \mathcal{D} t$ denotes the Jaumann time derivative defined by

$$
\frac{\mathcal{D} \boldsymbol{A}}{\mathcal{D} t}=\frac{\mathrm{D} \boldsymbol{A}}{\mathrm{D} t}+\boldsymbol{A} \cdot \boldsymbol{W}-\boldsymbol{W} \cdot \boldsymbol{A}
$$

where $A$ is an arbitrary tensor of order two, $W$ is the rotation velocity tensor and $\mathrm{D} / \mathrm{D} t$ is the material time derivative of $\boldsymbol{A}$. The three constants involved in the constitutive Equation (1), the zero-shear viscosity $\eta_{0}$, the relaxation time $\lambda_{1}$ and retardation time $\lambda_{2}$ of the bubble suspension, are given by

$$
\begin{gathered}
\eta_{0}=\eta_{\mathrm{c}}(1+\phi), \\
\lambda_{1}=\frac{6}{5}\left(\frac{\eta_{\mathrm{c}} R}{\sigma}\right)\left(1+\frac{16}{15} \phi\right), \\
\lambda_{2}=\frac{6}{5}\left(\frac{\eta_{\mathrm{c}} R}{\sigma}\right)\left(1-\frac{8}{5} \phi\right),
\end{gathered}
$$

where $\eta_{\mathrm{c}}$ is the shear viscosity of the liquid phase, $R$ is the bubble radius, $\sigma$ is the interface tension between liquid and gas phases and $\phi$ is the gas volume fraction. The relaxation time $\lambda_{1}$ and retardation time $\lambda_{2}$ for the system vary proportionally with the bubble radius $R$ and inversely proportionally with the interface tension $\sigma$. In a steady shear flow, the velocity vector is assumed to be 
$\boldsymbol{u}=\left(\dot{\gamma} x_{2}, 0,0\right)$, where $\dot{\gamma}$ is the constant shear rate. The Oldroyd model (1) - (5) gives expressions for the shear viscosity $\eta$ and the normal stress differences $N_{1}$ and $N_{2}$ of a bubble suspension in the form

$$
\begin{gathered}
\eta=\eta_{0}\left[\frac{1+\lambda_{\mathrm{lr}} \lambda_{2 \mathrm{r}}\left(\frac{6}{5} \mathrm{~N}_{\mathrm{Ca}}\right)^{2}}{1+\lambda_{\mathrm{lr}}^{2}\left(\frac{6}{5} \mathrm{~N}_{\mathrm{Ca}}\right)^{2}}\right], \\
N_{1}=2 \eta_{0} \dot{\gamma}\left[\frac{\left(\lambda_{\mathrm{lr}}-\lambda_{\mathrm{2r}}\right)\left(\frac{6}{5} \mathrm{~N}_{\mathrm{Ca}}\right)}{1+\lambda_{\mathrm{lr}}^{2}\left(\frac{6}{5} \mathrm{~N}_{\mathrm{Ca}}\right)^{2}}\right], \\
N_{2}=-\frac{1}{2} N_{1},
\end{gathered}
$$

where $\eta_{0 \mathrm{r}}=\eta_{0} / \eta_{\mathrm{c}}, \quad \lambda_{\mathrm{rr}}=\lambda_{1} / \lambda_{\mathrm{c}}$, and $\lambda_{2 \mathrm{r}}=\lambda_{2} / \lambda_{\mathrm{c}}$ are the relative zero-shear viscosity, the reduced relaxation time and reduced retardation time, respectively, and $\lambda_{\mathrm{c}}=6 \eta_{\mathrm{c}} R / 5 \sigma$ is the relaxation time of a single bubble. The capillary number is defined as $\mathrm{N}_{\mathrm{Ca}}=\eta_{\mathrm{c}} \dot{\gamma} R / \sigma \quad$ [11].

Pal [11] presented a rheological constitutive equation for concentrated suspensions containing bubbles, based on the Oldroyd model [2]. For a steady shear flow, the Equations (6)-(8) remain the same, but new improved expressions for the relative zero-shear viscosity, reduced relaxation time and reduced retardation time are derived using the differential effective medium approach. According to this approach, a concentrated suspension is obtained from an initial continuous phase by successively adding infinitesimal quantities of bubbles to the system until the final volume fraction of bubbles is reached. The increment changes in zero-shear viscosity, relaxation time and retardation time are calculated using the solution of a dilute system by treating the suspension as an equivalent effective medium that is homogeneous with respect to the new bubbles added. The differential equations derived in this manner are integrated to obtain the solutions for a concentrated suspension of bubbles. Following Krieger and Dougherty, the incremental increase in $\phi$ is taken to be $\mathrm{d} \phi /\left(1-\phi / \phi_{\mathrm{m}}\right)$, where $\phi_{\mathrm{m}}$ is an adjustable parameter, the maximum packing volume fraction of undeformed bubbles. This expression developed by Krieger and Dougherty takes account of the fact that voids between existing bubbles are created when new bubbles are dispersed in the suspension. For a random close packing of monodisperse spherical bubbles, $\phi_{\mathrm{m}}=0.637$ [11].

The maximum packing volume fraction of undeformed bubbles depends on the size distribution of initial undeformed bubbles. One way to take account for the effect of the bubble size distribution on the rheological properties of bubble suspensions is to include the parameter $\phi_{\mathrm{m}}$. Hence, the new equations for $\eta_{0}$, $\lambda_{1}$ and $\lambda_{2}$ [11] are given as

$$
\eta_{0}=\eta_{\mathrm{c}}\left(1-\frac{\phi}{\phi_{\mathrm{m}}}\right)^{-\phi_{\mathrm{m}}},
$$




$$
\begin{aligned}
& \lambda_{1}=\lambda_{\mathrm{c}}\left(1-\frac{\phi}{\phi_{\mathrm{m}}}\right)^{-(16 / 15) \phi_{\mathrm{m}}}, \\
& \lambda_{2}=\lambda_{\mathrm{c}}\left(1-\frac{\phi}{\phi_{\mathrm{m}}}\right)^{(8 / 5) \phi_{\mathrm{m}}} .
\end{aligned}
$$

\section{Analytical Method for Calculating Pipe Flows}

The pipe flow represents a fluid flow forced by a pressure difference through a pipe line. The most important flow is the steady laminar flow of a Newtonian liquid through a straight, circular pipe. The mathematical formulation is done in cylindrical coordinates $(r, \varphi, z)$. The fully developed flow is characterized by the fact that the velocity field consists only of the axial component $w(r)$ as a function of the radial variable $r$. Hence, the shear rate $\dot{\gamma}=\mathrm{d} w / \mathrm{d} r$ and the shear stress $\tau$ depend only on the radial variable $r$. The fully developed flow in a circular pipe is a balance between pressure forces and viscous forces [17] [18], i.e.

$$
\tau=-\frac{\Delta p}{2 L} r
$$

in which $\Delta p$ is the pressure difference along the pipe, $L$ is the pipe length and $R_{\mathrm{c}}$ is the pipe radius. The constitutive equation prescribes how the shear stress is related to the fluid velocity. A viscous fluid sticks at the pipe wall so that $w=0$ at $r= \pm R_{\mathrm{c}}$. Hence, a Newtonian fluid with shear viscosity $\mu$ has the velocity profile

$$
w(r)=W_{\max }\left[1-\left(\frac{r}{R_{\mathrm{c}}}\right)^{2}\right],
$$

with the maximal velocity $W_{\max }=\Delta p R_{\mathrm{c}}^{2} / 4 \mu L$ in the centerline of the pipe. The flow volume rate [17] [18] is given by

$$
Q=\frac{\pi R_{\mathrm{c}}^{4} \Delta p}{8 L \mu} .
$$

In the following, the steady laminar flow of a bubbly suspension is calculated using the Oldroyd model (1) with the new expressions (9)-(11) and $\phi_{\mathrm{m}}=0.637$. Based on the Equation (6), a cubic equation for the material behavior is obtained, i.e.

$$
\eta_{0} x(r) \cdot \frac{1+\lambda_{1} \lambda_{2} x^{2}(r)}{1+\lambda_{1}^{2} x^{2}(r)}=-\frac{\Delta p}{2 L} r,
$$

where the shear rate $x(r)$ is the function to be determined and $C:=\Delta p / 2 L$. Transformation gives the normal form of a cubic equation

$$
x^{3}+a x^{2}+b x+c=0,
$$

with the coefficients

$$
a=\frac{(C r) \lambda_{1}}{\eta_{0} \lambda_{2}}, \quad b=\frac{1}{\lambda_{1} \lambda_{2}}, \quad c=\frac{C r}{\eta_{0} \lambda_{1} \lambda_{2}} .
$$


By the substitution $x=z-a / 3$, the square term in Equation (16) is eliminated, and the reduced form of the cubic equation

$$
z^{3}+p z+q=0
$$

is obtained with coefficients

$$
\begin{gathered}
p=b-\frac{a^{2}}{3}, \\
\Rightarrow p(r)=\frac{3 \eta_{0}^{2} \lambda_{2}-(C r)^{2} \lambda_{1}^{3}}{3 \eta_{0}^{2} \lambda_{1} \lambda_{2}^{2}}, \\
q=\frac{2 a^{3}}{27}-\frac{a b}{3}+c, \\
\Rightarrow q(r)=\frac{2(C r)^{3} \lambda_{1}^{4}-9(C r) \eta_{0}^{2} \lambda_{1} \lambda_{2}+27(C r) \eta_{0}^{2} \lambda_{2}^{2}}{27 \eta_{0}^{3} \lambda_{1} \lambda_{2}^{3}} .
\end{gathered}
$$

The solutions of Equation (18) are calculated by Cardano's formula [19]. First, the substitution $z=u+v$ is performed. Comparison of the coefficients gives $-p=3 u v$ and $-q=u^{3}+v^{3}$. According to Vieta's theorem, $u^{3}$ and $v^{3}$ are the solutions of the quadratic equation $t^{2}+q t-\left(p^{3} / 27\right)=0$. Hence, the solutions of the reduced form of the cubic Equation (18) are obtained in the form

$$
\begin{gathered}
u=\sqrt[3]{-\frac{q}{2}+\sqrt{\Delta}}, \\
v=\sqrt[3]{-\frac{q}{2}-\sqrt{\Delta}},
\end{gathered}
$$

in which $\Delta$ is the discriminant:

$$
\Delta:=\left(\frac{q}{2}\right)^{2}+\left(\frac{p}{3}\right)^{3} .
$$

$\Delta$ determines the behavior of the solutions significantly, that is, depending on whether $\Delta$ is greater, smaller than or equal to zero, the solutions are calculated differently. The third roots $u$ and $v$ have to be chosen so that the condition $-p=3 u v$ is met. The analytical procedure can be summarized as follows:

$$
z= \begin{cases}u+v, & \text { if } \Delta>0 \\ 2 u, & \text { if } \Delta=0 \\ \text { equation (27) or (28), } & \text { if } \Delta<0\end{cases}
$$

The last case $\Delta<0$ depends on the sign of the solution. The physically correct solution is given by the fact that the velocity profile is continuously differentiable.

If $\Delta>0$, there is one real solution and two conjugated complex solutions. A solution can simply be determined by solving the formulas for $u$ and $\mathrm{V}$, and subsequent adding. The other solutions are obtained by multiplying $u$ and $v$ with the primitive third unit roots. Therefore, the following formulas for the solutions of the reduced cubic Equation (18) are to be specified [19]:

$$
z_{1}=u+v
$$




$$
\begin{aligned}
& z_{2}=\left(-\frac{1}{2}+i \frac{1}{2} \sqrt{3}\right) u+\left(-\frac{1}{2}-i \frac{1}{2} \sqrt{3}\right) v, \\
& z_{3}=\left(-\frac{1}{2}-i \frac{1}{2} \sqrt{3}\right) u+\left(-\frac{1}{2}+i \frac{1}{2} \sqrt{3}\right) v .
\end{aligned}
$$

If $\Delta=0$, it has to be distinguished which values $p$ and $q$ have, since the multiplicity of the solution depends on these values. If $p=q=0$, then $z_{1,2,3}=0$. If $p \neq q \neq 0$, then $u=v=-(q / 2)^{1 / 3}$. According to the formulas given in the Equations (24)-(26), there is a simple real solution and a double real solution: $z_{1}=2 u=(-4 q)^{1 / 3}=3 q / p$ and $z_{2,3}=-u=(q / 2)^{1 / 3}=-3 q / 2 p \quad$ [19].

If $\Delta<0$ (i.e. also $p<0$ ), the third roots $u$ and $v$ are conjugated complex to each other. Since $u+v=u+\bar{u}=2 \operatorname{Re}(u)$, three different real solutions are obtained. The third roots are determined using trigonometric functions. They are obtained in the form:

$$
\begin{gathered}
z_{1}=\left(\sqrt{-\frac{4}{3} p}\right) \cos \left(\frac{1}{3} \arccos \left(-\frac{q}{2} \sqrt{-\frac{27}{p^{3}}}\right)\right) \\
z_{2,3}=-\left(\sqrt{-\frac{4}{3} p}\right) \cos \left(\frac{1}{3} \arccos \left(-\frac{q}{2} \sqrt{-\frac{27}{p^{3}}}\right) \pm \frac{\pi}{3}\right) .
\end{gathered}
$$

The solutions of the original Equation (16) are determined by the re-substitution $x=z-a / 3$

The real solution is always selected. Rejecting the complex solutions can already be realized when determining $u$ and $v$. Unfortunately, there is no common procedure to determine the sign of a real solution in advance. However, note that the shear rate $x(r)$ decreases or increases from the pipe centerline to the pipe wall, that is $x(r)<0$ for $r>0$ and $x(r)>0$ for $r<0$. The discriminant $\Delta$ is positive in the example calculations with capillary number $\mathrm{Ca}<1$ while the discriminant also becomes negative in the example calculations with capillary number $\mathrm{Ca} \geq 1$. Based on the shear rate $x(r)$, the velocity profile is calculated by the composed trapezoidal rule, which is an integration method with accuracy order two [20] [21].

\section{Discussion of the Results}

As a reference example, the steady laminar pipe flow of a two-phase system consisting of an intermediate viscosity liquid and air bubbles is considered. Small, spherical, monodisperse air bubbles are dispersed in the liquid. The liquid is assumed to have a Newtonian rheology and the density and surface tension of water, that is $\rho_{\mathrm{c}}=1000 \mathrm{~kg} / \mathrm{m}^{3}$ and $\sigma=0.072 \mathrm{~N} / \mathrm{m}$ at $20^{\circ} \mathrm{C}$. The systems considered here are representative for food systems in industrial processes. The pressure difference along the pipe is postulated to be constant and moderate. The necessary material data and process data are given in Table 1 and Table 2.

The dimensionless numbers, the Reynolds number Re and capillary number $\mathrm{Ca}[22]$, to characterize the pipe flow of a suspension of bubbles are defined as 
Table 1. Material data of the liquid for the two-phase systems at $20^{\circ} \mathrm{C}$.

\begin{tabular}{ccc}
\hline Material quantity & Value & Unity \\
\hline Density $\rho_{\mathrm{c}}$ & 1000 & $\mathrm{~kg} / \mathrm{m}^{3}$ \\
Shear viscosity $\eta_{\mathrm{c}}$ & 1 & $\mathrm{Pas}$ \\
Surface tension $\sigma$ & 0.072 & $\mathrm{~N} / \mathrm{m}$ \\
\hline
\end{tabular}

Table 2. Process data for the two-phase systems at $20^{\circ} \mathrm{C}$.

\begin{tabular}{ccc}
\hline Process quantity & Value & Unity \\
\hline Temperature $\mathcal{O}$ & 20 & ${ }^{\circ} \mathrm{C}$ \\
Pipe length $L$ & 1 & $\mathrm{~m}$ \\
Pipe radius $R_{\mathrm{c}}$ & 0.025 & $\mathrm{~m}$ \\
Pressure difference $\Delta p$ & 2500 & $\mathrm{~Pa}$ \\
Bubble radius $R$ & $0.0001 ; 0.0005 ; 0.002$ & $\mathrm{~m}$ \\
Gas volume fraction $\phi$ & $0.15 ; 0.30 ; 0.45 ; 0.50$ & $\mathrm{~m}^{3} / \mathrm{m}^{3}$ \\
Parameter $\phi_{\mathrm{m}}$ & 0.637 & $\mathrm{~m}^{3} / \mathrm{m}^{3}$ \\
\hline
\end{tabular}

$$
\begin{gathered}
\mathrm{Re}=\frac{Q}{v R_{\mathrm{c}}}, \\
\mathrm{Ca}=\frac{\eta_{\mathrm{c}} \dot{\gamma}\left(R_{\mathrm{c}}\right) R}{\sigma},
\end{gathered}
$$

where $Q$ is the volume flow rate, $v=\eta\left(\dot{\gamma}\left(R_{\mathrm{c}}\right)\right) / \rho$ is the kinematic viscosity of the bubble suspension and $\dot{\gamma}\left(R_{\mathrm{c}}\right)$ is the shear rate at the pipe wall. The density of the suspension is given by $\rho=(1-\phi) \cdot \rho_{\mathrm{c}}+\phi \cdot \rho_{\mathrm{g}}$ with the air density $\rho_{\mathrm{g}}=1.2 \mathrm{~kg} / \mathrm{m}^{3}$ at $20^{\circ} \mathrm{C}$. Llewellin, Mader, Wilson and Manga [7] [8] [9] investigated already the steady laminar pipe flow of bubbly liquids, based on the Frankel and Acrivos model [5] [6]. The results presented in this article are based on the modified Oldroyd model [1] [2] [3] [4] [10] [11]. They were determined by Cardano's formula.

The bubble radius $R$ of initial undeformed monodisperse bubbles is varied, and the effect on the rheological behavior of suspensions is studied. In Figures $1-3$, the velocity profiles of the two-phase systems with gas volume fraction $\phi=$ 0.15 (red solid line), $\phi=0.30$ (black solid line), $\phi=0.45$ (green solid line), and $\phi$ $=0.50$ (blue solid line) are shown. Additionally, the velocity profiles of the corresponding Newtonian two-phase systems (dashed line) are shown for purposes of comparison. The Newtonian systems differ from the non-Newtonian systems in that the shear viscosity $\eta$ is replaced by the constant zero-shear viscosity $\eta_{0}$ and the volume flow rate $Q$ is calculated according to formula (14). Figure 1 and Figure 2 are examples for pipe flows with a small capillary number $\mathrm{Ca}<1$, whereas Figure 3 is an example for pipe flows with an intermediate capillary number $\mathrm{Ca} \geq 1$

At small capillary numbers $\mathrm{Ca}<1$, suspensions of bubbles in a pipe line show a parabolic velocity profile indicating a Newtonian rheology [8]. Suspensions 


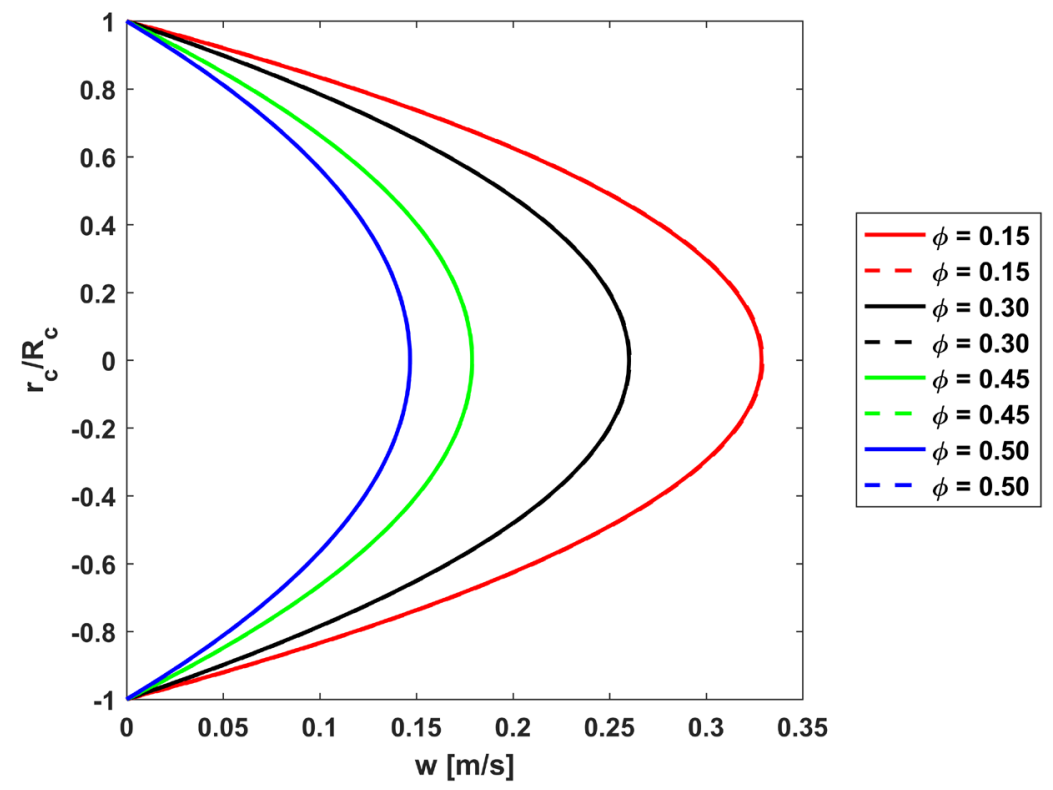

Figure 1. Velocity profiles of two-phase systems with different gas volume fractions $\phi$. The shear viscosity of the liquid is $\eta_{\mathrm{c}}=1$ Pas, and the bubble radius is $R$ $=0.0001 \mathrm{~m}$. The dashed lines show the velocity profiles of the corresponding two-phase systems, which have a Newtonian rheology.

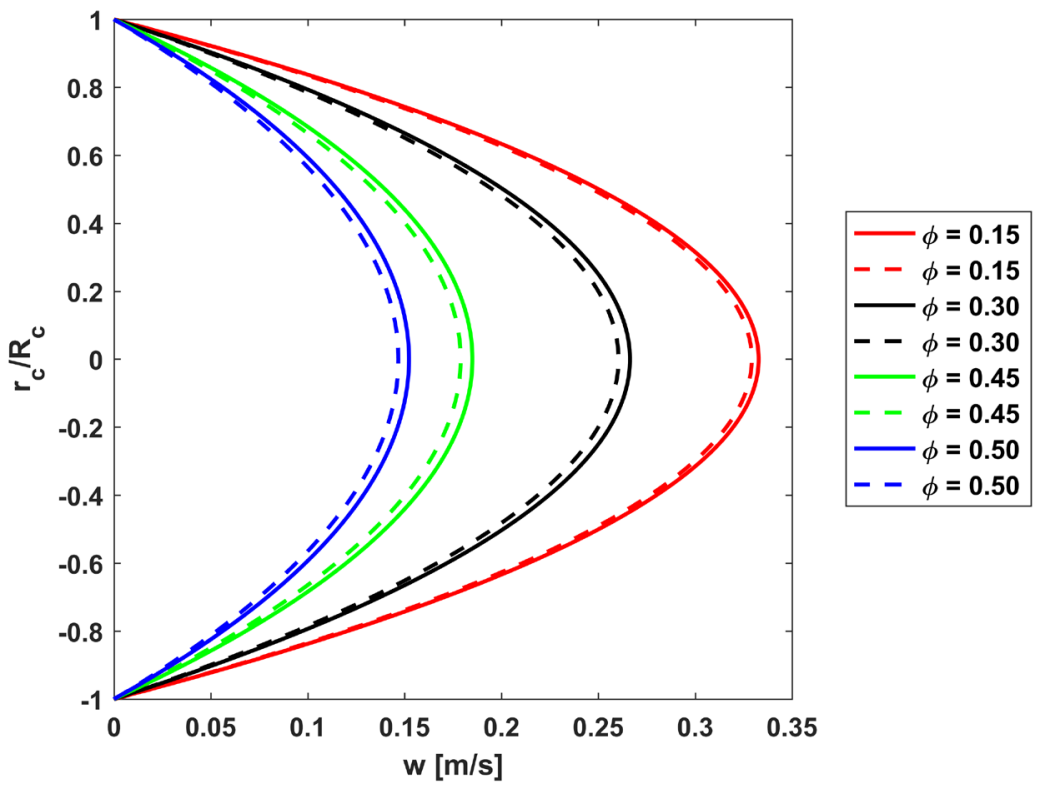

Figure 2. Velocity profiles of two-phase systems with different gas volume fractions $\phi$. The shear viscosity of the liquid is $\eta_{\mathrm{c}}=1 \mathrm{Pas}$, and the bubble radius is $R$ $=0.0005 \mathrm{~m}$. The dashed lines show the velocity profiles of the corresponding two-phase systems, which have a Newtonian rheology.

with a low gas volume fraction flow faster along the pipe than suspensions with a high gas volume fraction. At intermediate capillary numbers $\mathrm{Ca} \geq 1$, the suspensions of bubbles deviate strongly from the corresponding Newtonian systems. As explained by Llewellin, Mader and Wilson [7] [8] [9], the suspension shows two 


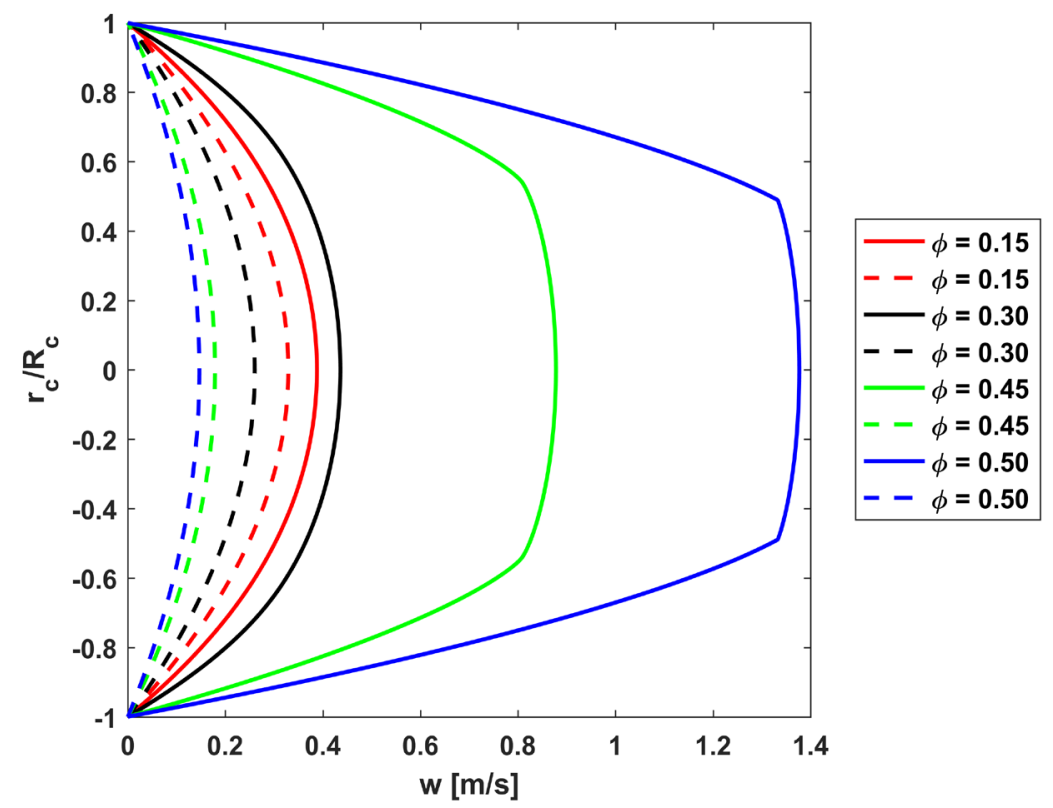

Figure 3. Velocity profiles of two-phase systems with different gas volume fractions $\phi$. The shear viscosity of the liquid is $\eta_{\mathrm{c}}=1 \mathrm{Pas}$, and the bubble radius is $R$ $=0.002 \mathrm{~m}$. The dashed lines show the velocity profiles of the corresponding two-phase systems, which have a Newtonian rheology.

regimes of flow, an inner plug flow where deformation rates are low and an outer flow where deformation rates are high. In this case, a relatively undeformed plug is surrounded by a rapidly deforming outer flow [7] [8] [9]. Suspensions with a high gas volume fraction flow now faster along the pipe than suspensions with a low gas volume fraction. The reason is that, at intermediate capillary numbers $\mathrm{Ca} \geq 1$, air bubbles are stretched and form free slip surfaces in the outer flow. As a result, the shear viscosity $\eta$ in the suspension is decreased and the Reynolds number Re is increased significantly. The effect is more strongly, the greater the gas volume fraction $\phi$ is. If $\mathrm{Ca}>1$, a suspension of bubbles has again a parabolic velocity profile. The Reynolds number Re, however, increases now with the gas volume fraction $\phi$.

The material behavior is explained by Figure 4 . This figure shows the relative viscosity $\eta_{r}=\eta / \eta_{\mathrm{c}}$ predicted from the modified Oldroyd model (1) with the new expressions (9)-(11) as a function of the capillary number $\mathrm{Ca}$ at the values of $\phi$ given above. As analyzed by Pal [11], the plot exhibits three different regions: constant viscosity region at low values of $\mathrm{Ca}$, decreasing viscosity at intermediate values of $\mathrm{Ca}$ and constant viscosity region at high values of $\mathrm{Ca}$. The relative viscosity $\eta_{r}$ is greater than unity at capillary numbers $\mathrm{Ca}<1$ and is less than unity at capillary numbers $\mathrm{Ca}>1$. With the increase in $\phi$, the relative viscosity $\eta_{r}$ increases at $\mathrm{Ca}<1$ and decreases at $\mathrm{Ca}>1$. Llewellin, Mader and Wilson [7] [8] [9] explained this viscosity behavior physically: For small capillary numbers $\mathrm{Ca}<1$, bubbles are approximately spherical so that flow lines in the suspension are distorted. As a result, the viscosity of the suspension increases. 


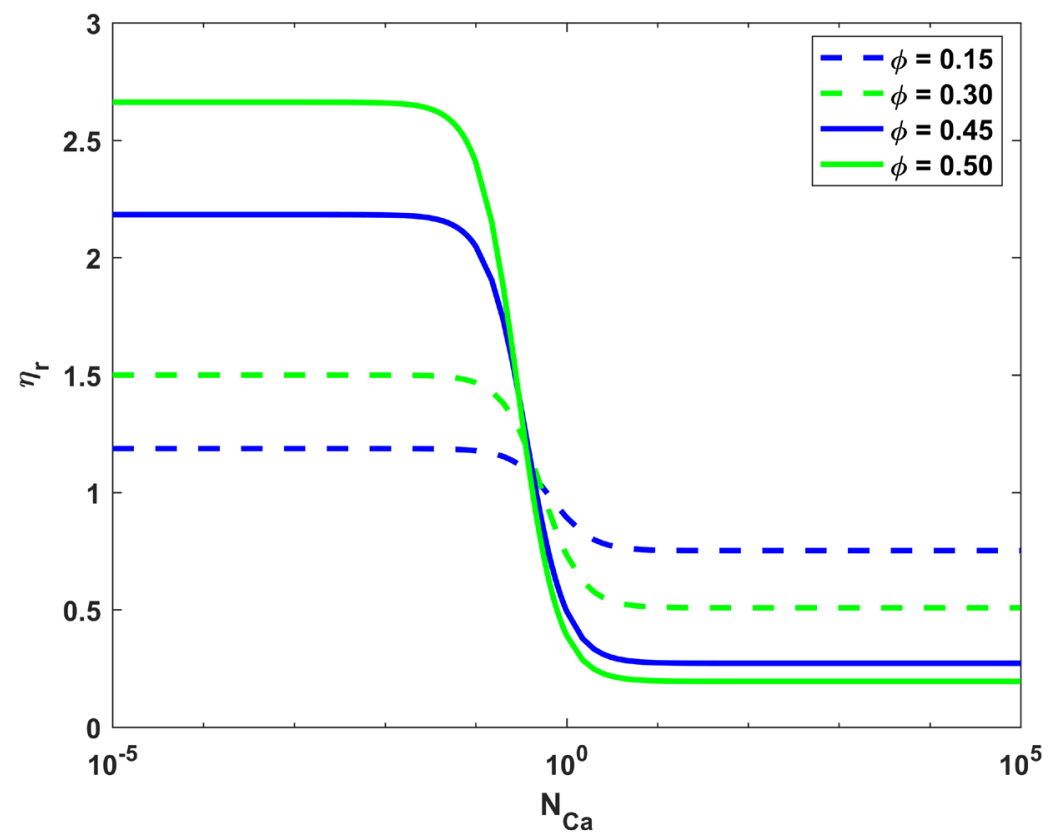

Figure 4. Relative viscosity $\eta_{\mathrm{r}}$ as a function of the capillary number Ca predicted by the modified Oldroyd model (1) with the new expressions (9)-(11). The predictions are shown for different values of the gas volume fraction $\phi$. The value of $\phi_{\mathrm{m}}=0.637$ corresponds to a random close packing of uniform spherical bubbles [10] [11].

For intermediate or high capillary numbers $\mathrm{Ca} \geq 1$, bubbles are stretched so that they form free slip surfaces in the suspension, and the viscosity decreases.

If the capillary number $\mathrm{Ca}$ is less than unity, the Reynolds number Re decreases with increasing gas volume fraction. If the capillary number $\mathrm{Ca}$ is greater than unity, the opposite effect occurs, that is, the Reynolds number Re increases with the gas volume fraction. In Tables 3-5, the values of relevant material and process quantities relating to Figures 1-3 are specified: the capillary number $\mathrm{Ca}$, the Reynolds number Re, the shear viscosity $\eta\left(\dot{\gamma}\left(R_{\mathrm{c}}\right)\right)$ and shear rate $\dot{\gamma}\left(R_{c}\right)$ at the pipe wall, the volume flow rate $Q$ as well as the velocity $w(0)$ in the pipe centerline. Relevant results relating to the rheological behavior of suspensions containing bubbles are also summarized in the articles [23] [24], where the Frankel and Acrivos model is briefly explained.

\section{Conclusion}

The steady laminar pipe flow of a suspension with a gas volume fraction $\leq 0.5$ and small or intermediate bubble deformations is calculated in long, and straight sections of a circular pipe. The calculations are based on the constitutive equation that was originally derived by Oldroyd for dilute emulsions and further developed by Pal for concentrated suspensions of bubbles. The pipe flows are characterized by a Reynolds number and a capillary number defined in this article. If the capillary number is less or greater than unity, the suspension of bubbles shows a Newtonian rheology. If the capillary is approximately unity, the 
Table 3. Relevant data relating to Figure $1, R=0.0001 \mathrm{~m}$.

\begin{tabular}{ccccc}
\hline Quantity & $\phi=0.15$ & $\phi=0.30$ & $\phi=0.45$ & $\phi=0.50$ \\
\hline Ca & 0.0366 & 0.0290 & 0.0199 & 0.0164 \\
Re & 9.2420 & 4.7698 & 1.7727 & 1.0848 \\
$\eta\left(\gamma^{\prime}\left(R_{\mathrm{c}}\right)\right)[\mathrm{Pas}]$ & 1.1853 & 1.4973 & 2.1773 & 2.6540 \\
$\gamma\left(R_{\mathrm{c}}\right)\left[\mathrm{s}^{-1}\right]$ & 26.3640 & 20.8707 & 14.3524 & 11.7748 \\
$Q\left[\mathrm{~m}^{3} / \mathrm{s}\right]$ & 0.000322132 & 0.000254937 & 0.000175271 & 0.000143782 \\
$\omega(0)[\mathrm{m} / \mathrm{s}]$ & 0.3287 & 0.2601 & 0.1788 & 0.1467 \\
\hline
\end{tabular}

Table 4. Relevant data relating to Figure $2, R=0.0005 \mathrm{~m}$.

\begin{tabular}{ccccc}
\hline Quantity & $\boldsymbol{\phi}=\mathbf{0 . 1 5}$ & $\boldsymbol{\phi}=\mathbf{0 . 3 0}$ & $\boldsymbol{\phi}=\mathbf{0 . 4 5}$ & $\boldsymbol{\phi}=\mathbf{0 . 5 0}$ \\
\hline $\mathrm{Ca}$ & 0.1876 & 0.1520 & 0.1069 & 0.0883 \\
$\mathrm{Re}$ & 9.6227 & 5.1628 & 1.9898 & 1.2322 \\
$\eta\left(\gamma\left(R_{\mathrm{c}}\right)\right)[\mathrm{Pas}]$ & 1.1570 & 1.4275 & 2.0301 & 2.4565 \\
$\gamma\left(R_{\mathrm{c}}\right)\left[\mathrm{s}^{-1}\right]$ & 27.0099 & 21.8912 & 15.3932 & 12.7212 \\
$Q\left[\mathrm{~m}^{3} / \mathrm{s}\right]$ & 0.000327379 & 0.000263077 & 0.000183430 & 0.000151160 \\
$w(0)[\mathrm{m} / \mathrm{s}]$ & 0.3327 & 0.2663 & 0.1850 & 0.1523 \\
\hline
\end{tabular}

Table 5. Relevant data relating to Figure $3, R=0.0020 \mathrm{~m}$.

\begin{tabular}{ccccc}
\hline Quantity & $\boldsymbol{\phi}=\mathbf{0 . 1 5}$ & $\boldsymbol{\phi}=\mathbf{0 . 3 0}$ & $\boldsymbol{\phi}=\mathbf{0 . 4 5}$ & $\boldsymbol{\phi}=\mathbf{0 . 5 0}$ \\
\hline $\mathrm{Ca}$ & 0.9641 & 1.3410 & 2.8733 & 4.1791 \\
$\mathrm{Re}$ & 15.0029 & 21.2071 & 79.0661 & 160.6685 \\
$\eta\left(\gamma\left(R_{c}\right)\right)[\mathrm{Pas}]$ & 0.9004 & 0.6473 & 0.3021 & 0.2077 \\
$\gamma\left(R_{c}\right)\left[\mathrm{s}^{-1}\right]$ & 34.7069 & 48.2766 & 103.4396 & 150.4483 \\
$Q\left[\mathrm{~m}^{3} / \mathrm{s}\right]$ & 0.000397227 & 0.000490020 & 0.001084688 & 0.001666643 \\
$\omega(0)[\mathrm{m} / \mathrm{s}]$ & 0.3878 & 0.4359 & 0.8777 & 1.3766 \\
\hline
\end{tabular}

suspension shows two regimes of flow, an inner plug flow and a rapidly deforming outer flow. These results were already found out by Llewellin, Mader, Wilson and Manga, who used the Frankel and Acrivos model. They explained different velocity profiles by bubble deformations depending on the capillary number. In this article, an analytical method and a physical more sensible model were used to determine solutions for pipe flows of suspensions containing bubbles more accurately. Furthermore, it can be shown: If the capillary number is less than unity, the Reynolds number decreases with increasing gas volume fraction. If the capillary number is equal to or greater than unity, the Reynolds number increases with increasing gas volume fraction.

\section{Acknowledgements}

This research project was supported by the German Ministry of Economics and 
Technology and the FEI (Forschungskreis der Ernährungsindustrie e.V., Bonn). Project AiF 17125 N. This project is part of the cluster "Proteinschäume in der Lebensmittelproduktion: Mechanismenaufklärung, Modellierung und Simulation" funded by the FEI and the AiF.

\section{Conflicts of Interest}

The authors declare no conflicts of interest regarding the publication of this paper.

\section{References}

[1] Oldroyd, J.G. (1950) On the Formulation of Rheological Equations of State. Proceedings of the Royal Society of London A, 200, 523-541. https://doi.org/10.1098/rspa.1950.0035

[2] Oldroyd, J.G. (1953) The Elastic and Viscous Properties of Emulsions and Suspensions. Proceedings of the Royal Society of London A, 218, 122-132. https://doi.org/10.1098/rspa.1953.0092

[3] Oldroyd, J.G. (1958) Non-Newtonian Effects in Steady Motion of Some Idealized Elastico-Viscous Liquids. Proceedings of the Royal Society of London A, 245, 278-297. https://doi.org/10.1098/rspa.1958.0083

[4] Fröhlich, H. and Sack, R. (1946) Theory of the Rheological Properties of Dispersions. Proceedings of the Royal Society of London A, 185, 415-430. https://doi.org/10.1098/rspa.1946.0028

[5] Frankel, N.A. and Acrivos, A. (1970) The Constitutive Equation for a Dilute Emulsion. Journal of Fluid Mechanics, 44, 65-78. https://doi.org/10.1017/S0022112070001696

[6] Cox, R.G. (1969) The Deformation of a Drop in a General Time-Dependent Fluid flow. Journal of Fluid Mechanics, 37, 601-623. https://doi.org/10.1017/S0022112069000759

[7] Llewellin, E.W., Mader, H.M. and Wilson, S.D.R. (2002) The Rheology of a Bubbly liquid. Proceedings of the Royal Society of London A, 458, 987-1016. https://doi.org/10.1098/rspa.2001.0924

[8] Llewellin, E.W., Mader, H.M. and Wilson, S.D.R. (2002) The Constitutive Equation and Flow Dynamics of Bubbly Magmas. Geophysical Research Letters, 29, 23-1-23-4. https://doi.org/10.1029/2002GL015697

[9] Llewellin, E.W. and Manga, M. (2005) Bubble Suspension Rheology and Implications for Conduit Flow. Journal of Volcanology and Geothermal Research, 143, 205-217. https://doi.org/10.1016/j.jvolgeores.2004.09.018

[10] Pal, R. (2003) Rheological Behavior of Bubble-Bearing Magmas. Earth and Planetary Science Letters, 207, 165-179. https://doi.org/10.1016/S0012-821X(02)01104-4

[11] Pal, R. (2004) Rheological Constitutive Equation for Bubbly Suspensions. Industrial \& Engineering Chemistry Research, 43, 5372-5379. https://doi.org/10.1021/ie040011r

[12] Pal, R. (1992) Rheological Properties of Emulsions of Oil in Aqueous Non-Newtonian Polymeric Media. Chemical Engineering Communications, 111, 45-60. https://doi.org/10.1080/00986449208935980

[13] Pal, R. (1996) Viscoelastic Properties of Polymer-Thickened Oil-in-Water Emulsions. Chemical Engineering Science, 51, 3299-3305. 
https://doi.org/10.1016/0009-2509(95)00393-2

[14] Rust, R.C. and Manga, M. (2002) Effects of Bubble Deformation on the Viscosity of Dilute Suspensions. Journal of Non-Newtonian Fluid Mechanics, 104, 53-63. https://doi.org/10.1016/S0377-0257(02)00013-7

[15] Stein, D.J. and Spera, F.J. (2002) Shear Viscosity of Rhyolite-Vapor Emulsions at Magmatic Temperatures by Concentric Cylinder Rheometry. Journal of Volcanology and Geothermal Research, 113, 243-258. https://doi.org/10.1016/S0377-0273(01)00260-8

[16] Choi, S.J. and Schowalter, W.R. (1975) Rheological Properties of Nondilute Suspensions of Deformable Particles. Physics of Fluids, 18, 420-427. https://doi.org/10.1063/1.861167

[17] Böhme, G. (2000) Strömungsmechanik nichtnewtonscher Fluide. Leitfäden der angewandten Mathematik und Mechanik. B. G. Teubner, Stuttgart. https://doi.org/10.1007/978-3-322-80140-1

[18] Giesekus, H. (1994) Phänomenologische Rheologie. Eine Einführung. Springer-Verlag, Berlin, Heidelberg, New York. https://doi.org/10.1007/978-3-642-57953-0

[19] Vorderwülbecke, S. (2015) Exakte und effiziente Berechnung der positiven, reellen Nullstellen von Polynomen bis zu vierten Grades für statistische (Monte Carlo) Simulation von Flugbahnen mit algebraisch beschriebenen Hindernissen. Seminararbeit. Studiengang Scientific Programming. Fachhochschule Aachen, Campus Jülich.

[20] Stoer, J. (1999) Numerische Mathematik 1. Springer-Verlag, Berlin, Heidelberg, New York. https://doi.org/10.1007/978-3-662-09021-3

[21] Stoer, J. and Bulirsch, R. (2000) Numerische Mathematik 2. Springer-Verlag, Berlin, Heidelberg, New York. https://doi.org/10.1007/978-3-662-09025-1

[22] Spurk, J.H. (1992) Dimensionsanalyse in der Strömungsmechanik. Springer-Verlag, Berlin, Heidelberg, New York. https://doi.org/10.1007/978-3-662-01581-0

[23] Gladbach, K., Delgado, A. and Rauh, C. (2014) Modelling and Simulation of the Transport of Protein Foams. Proceedings in Applied Mathematics and Mechanics, 14, 859-860. https://doi.org/10.1002/pamm.201410410

[24] Gladbach, K., Delgado, A. and Rauh, C. (2016) Modelling and Simulation of Food Foams. Proceedings in Applied Mathematics and Mechanics, 16, 595-596. https://doi.org/10.1002/pamm.201610286 